THURSDAY, AUGUST 10, 1871

\section{THE ORGANISATION OF LOCAL SCIENTIFIC EFFORT}

A MONG the many topics of national importance A which have been discussed at the recent meeting of the British Association, there is none which promises to bear more fruit, or which. we more gladly bring before the notice of our readers, than a scheme already suggested in these columns, which has been discussed and adopted at a full and influential meeting of representatives of all branches of Science, the President of the Association, Sir William Thomson, being in the chair.

This scheme is essentially as follows: $-\mathrm{It}$ is proposed in the first instance, to make an attempt to extend and improve the present system of giving scientific lectures to the people, and by this means to avaken an interest in science and scientific progress in places where otherwise there would be little probability of such good work being done.

There is little need that we should expatiate on the extreme importance of this object, and on the value of the results which are certain to follow from an energetic carrying out of the proposal. With the example of Manchester and other large towns before us, it is not too much to hope that as soon as the scheme is properly developed, the beneficial effects already experienced in these places wlll become general throughout the country. In Man. chester, to take one instance, we find that each Science Lecture has, on an average, been attended by upwards of one thousand persons, and that the interest excited by the lectures has not been a mere temporary amusement is evidenced by the fact that the lectures when reprinted have sold by tens of thousands. In Belfast, also, Science Lectures to working-men have been most successfully given for more than ten years. In this way it is clear that not merely the auditors, but a very large outside public, have benefited by this method of bringing science and its teachings home to everyone. A project, which has been so successful over limited areas, and which must be as successful if tried on a larger scale, is well deserving of being adopted and extended by so important a body as the British Association.

There is another consideration which renders the adoption of this scheme by the British Association doubly valuable. The danger attending the delivery of popular lectures has always been that true scientific method may be lost sight of in the desire of the lecturer to merely please the eye, or to keep up interest in the auditory by mere sensational display. It is to be hoped that we shall now have a guarantee at any rate against this evii. It is not possible always to make science amusing, but we now possess ample experience which goes to show that a scientific lecture delivered by a competent man, fully impressed himself with the dignity of what he is cloing, is able to awake the interest and rivet the attention of those classes for whom the lectures are specially intended.

This, however, after all, is only one side of the project. We do not for one moment wish to undervalue the ex- treme importance of science lectures, but we must not forget that they will have missed their mark if they have not engendered the desire for something more durable (because more useful) in the way of scientific instruction, which can be obtained in a variety of ways, as, for instance, in Mechanics' Institutions, in the science classes of the Science and Art Department, or in other organisations which may be subsequently developed.

It is not, however, merely a question of scientific instruction. Throughout the country we find societies, field clubs, local museums, \&c., all of which are more or less actively engaged in the pursuit of knowledge, local inquiries, or exploration, and all of which are working, more or less, at a disadvantage, in consequence of the chaotic state of our scientific arrangements, and from their lack of that power which springs from unity.

Now is ic too much to expect that under the best possible conditions such engines of scientific advancement would be more useful than they are at present, or that there would be more of them? We have only to look at what has been done in some of the higher schools even, to satisfy ourselves upon this point. At Rugby, Clifton, Marl. borough, not to mention other schools, we have museums and natural history societies existing side by side with the work of the school, and the masters testify in the most definite manner to the extreme importance of the culture obtained by such means. Now, if this is important for a limited number of schoolboys, how much more important must it be throughout the length and breadth of the land; where at present we find teaching going on without museums, museums existing in localities where there is no one to look after them, field clubs examining every inch of the ground, while a much richer region elsewhere is entirely unexplored, each worker, as it were, away from his support, and the workers few. It is as if an army were moving through a hostile country without commancer, without plan, without any power of combination, and without either vanguard, Uhlan, or second line.

Here then we have plainly before us the ground to be viewed by the Committee to which we have referred, a Committee which we doubt not will be appointed by the British Association with full power to reportupon, and, if necessary, to carry out at once, any measures which it may be desirable to take in the directions we have indicated. When once such a body is established, and its existence generally known, its work will soon take the most concrete form, a more concrete one than we have ventured to assign to it in this article; but it is clear that if limited in its functions in the first instance to the lecture arrangements to which we have referred, and to inquiries into the actual geographical position of and condition of our local societies, museums, field clubs, and the like, so that the committee should become the head-quarters of information on these subjects to those who wish to establish similar institutions in new districts, or to expand an existing one, the greatest possible good to science will follow. But it is not too much to hope that such a body would in time become the centre of influence as well as of information, would be able to mould actual and potential institutions into the best form for effective work, and would be able to economise their resources, and to increase the utility of each of them. 